\title{
Die neue Kantausgabe: Kants Briefwechsel.
}

\author{
Von Dr. Ernst Sänger.
}

Dank der ausserordentlich eifrigen und von seltener Gewissenhaftigkeit getragenen Bemühung Rudolf Reickes ist die Neue Kantausgabe im Juli um den dritten Band des Kantischen Briefwechsels bereichert worden. Über den ersten Band desselben ist KSt. V, 73-115, über den zweiten KSt. VI, 41-72, Bericht erstattet worden. Die nachfolgenden. Zeilen wollen auf das Wichtige und Interessante hinweisen, was, abgesehen von dem schon aus der 2. Hartensteinschen Ausgabe Bekannten, der dritte Band uns bietet. Derselbe enthält den privaten Briefwechsel der Jahre 1795-1803 samt einigen undatierten und einer Reihe nachgetragener Briefe, ferner Kants öffentliche und handschriftliche Erklürungen samt seinem letzten Willen, ausserdem sechs von Kant gedichtete Denkverse zu Ehren verstorbener Kollegen und drej Gedichte, die Kant von seinen Zuhörern gewidmet sind, schliesslich zehn von Kant abgefasste Stammbuchverse und eine Auswahl des Kantischen amtlichen Schriftverkehrs. Der Band weist nicht ganz die gleiche Stärke auf wie die beiden ersten; die neunjährige Privatkorrespondenz, welche er veröffentlicht, ist also von erheblich geringerem Umfang (ca. 350 Seiten) als die bloss sechsjährige, aber über 500 Seiten umfassende des vorhergehenden Bandes. Der Grund hierfür liegt auf der Hand: Kants Alter, seine andauernde Krankheit - diese beiden Dinge berührt er in den meisten seiner Briefe und damit verbunden eine Abnahme seiner geistigen Kräfte, geboten von selbst eine allmähliche Beschränkung seines Briefverkehrs. Wir wissen bloss von zwei Briefen Kants aus dem Jahre 1801, ferner bloss von sechs aus dem Jahre 1802 und bloss von einem aus dem Jahre 1803. Aus demselben Grunde erklärt sich, dass Kants Briefe spätestens vom Jahre 1800 ab in wissenschaftlicher Hinsicht von keiner Bedeutung sind.

Bevor ich auf die eigentliche Privatkorrespondenz dieses Bandes eingehe, gebe ich kurz an, um was es sich in den wichtigsten nachgetragenen Briefen (865-881) handelt. Dieselben gehen bis in den Dezember 1772 zurück. In diese Zeit fällt eine kurze Mitteilung Wielands des damaligen Weimarischen Hofrates, welcher Kant „Nachrichten an das Publikum" mit der Bitte, dieselben in weiten Kreisen bekannt zu machen, übersendet. Das 10. Exemplar soll Kant als Entschädigung für seine „Collecteur"-Dienste behalten (865). - Vom Sept. 1775 her datiert sich ein 
Schreiben von H. Chr, Boie und Chr. W. Dohm. Dis beiden aus der Litteraturgeschichte bekannten Männer haben die Absicht, unter dem Titel "Dentsches Museum" ein encyklopädisches Nationaljournal nach einem fehlgeschlagenen Versuche neu herauszugeben. Sie teilen im Überblick mit, welche Gegenstände das Journal, dessen Ziel sie in der Darbietung einer „unterhaltenden Gelehrsamkeit" sehen, behandeln soll, und ersuchen Kant um Mitarbeit an demselben (868). - Im Dez.1777 gelangt an Kant ein geheimer Aufruf zum Eintritt in die „Deutsche Union der Zwei und Zwa nziger". "Eine Gesellschaft von 22, theils Staatsmännern, theils offentlichen Lehrern, theils Privatpersonen, hat sich bereits über einen seit anderthalb Jahren in Vorschlag gebrachten Plan vereinigt, welcher ihrem Bedünken nach ein untrügliches und durch keine menschliche Macht zu hinderndes Mittel enthält, die Aufklärung und Bildung der Menschheit zu befördern und alle bisherigen Hindernisse derselben nach und nach zu zerstören." Der Aufruf ist "An die Freunde der Vernunft, der Wahrheit und der Tugend" gerichtet (868). - Bemerkenswert ist Kants Brief an Abraham Jacob Penzel vom Aug. 1777 wegen einiger „ohne genaue Prüfung nur so dahin geworfener" Notizen über den Vorzug der Zahl 9 bei einzelnen Völkern. „Es scheint mir dass ein dem ersten Ansehen nach so unerheblicher Umstand als die Übereinstimmung einiger Völker in dem Vorzuge einer Zahl und der Zusammenhang derselben mit der ältesten astronomie einen ziemlichen Fingerzeig auf die erste Schule der Wissenschaften der alten nationen abgeben könne. Vielleicht hat auch die Uralte obzwar geheim gehaltene Erfindung mit 9 Ziffern u. einer 0 zu rechnen zum Ansehen der Zahl 9 viel beygetragen" (869). - Von speziell philosophischem Interesse ist ein Brief des Geh. Sekretärs Aug. Wilh. Rehberg (vgl. den beiderseitigen früheren Briefwechsel), der an dem der 2. Aufl. der Kr. d. r. V. entnommenen Satze Kritik übt: „Mathematische Sätze werden aus der Anschauung und nicht aus dem Verstandesbegriffe gezogen" und dann Fragen aufwirft wie folgende: "Wie geht es zu, dass der Verstand bei der Erzeugung der Zahlen, welches ein reiner Actus seiner Spontaneität ist, an die synthetischen Sätze'der Arithmetik und Algebra gebunden ist? Warum kann er, der Zahlen willkührlich hervorbringt, keine $\sqrt{2}$ in Zahlen denken? da ihn doch die Natur der Form der Sinnlichkeit nicht verhindert, so wie die Natur des Raumes ihn hindert gerade Linien zu denken, die gervissen krummen gleich wären" (877). Die Reihe der nachgetragenen Briefe beschliesst der Anfang zu 566, einem Briefe des Tübinger Theologie-Professors Joh. Friedr. Flatt, der über einige Fragen der kritischen Philosophie um Aufklärung bittet.

Die eigentliche Privatkorrespondenz des dritten Bandes umfasst, wie bemerkt, die Jahre 1795-1803. Soweit die Briefe aus diesen Jahren an Kant gerichtet sind - das ist ja der bei weitem grössere Teil der gesammelten Briefe - , sind sie beredte Zeugen der unbegrenzten Achtung und Liebe, die Kant damals genossen hat. Sie stellen den lebenden Kant auf dem Höhepunkte seines Ruhmes dar. Kant ist für seine Zeit der Philosophenfürst geworden. Er wird umschmeichelt und verehrt. Verehrung wird ihm nicht bloss von seinen Schülern und seinen Kollegen gezollt, sondern in alle gebildeten Kreise ist sein Ruf gedrungen und hat 
ihm dort treue Anhänger gewonnen. "Wie sehr Sie theuerster Herr Professor," so schreibt Jachmann, "von der Danziger Kaufmannschaft verehrt werden, das habe ich zu meiner grössten Freude erfahren. Jedermann wünschte Ihnen gefällig werden zu können" (832). "Sie glauben nicht," so berichtet Conrad Stang aus Würzburg, "wie enthusiastisch Mädchen und Frauen für Ihr Sistem eingenommen sind, und wie allgemein diese wünschen, es zu kennen. Hier in Würzburg kömmt man in viele Frauenzimmergesellschaften, wo man sich beeifert, vor andern mehr Kenntniss Thres Sistems zu zeigen, und wo es stets das Lieblingsgespräch ausmacht. $\mathrm{Ja}$, was gewiss seltene Erscheinung ist, man hält sich nicht allein in den Schranken des praktischen Theiles, sondern wagt sich auch in das theoretische" (680). Ein russischer Reiteroffizier, Freiherr von Ungern-Sternberg, schreibt an und von Kant: „obgleich ich viele Helden gesehn habe, so kannte ich doch nicht den, welcher sein und das kommende Zeitalter iiberwunden hat (669).

Der Ruhm Kants ist es, der die Herausgeber wissenschaftlicher Zeitschriften veranlasst, sich mit der Bitte um Beiträge an Kant $z u$ wenden. Wir haben schon aus einem der nachgetragenen Briefe (868) ersehen, dass Kant zur Mitarbeit am "Deutschen Museum" aufgefordert wird. Wir sehen ferner, dass er um Beiträge für die Annalen (632), für die Horen (617), für das Journal der Professorin Mèreau in Jena (654), für den Kosmopolit (675) angegangen wird. Weiterhin erbittet sich Hufeland die Bearbeitung über „den so interessanten medezinischen Gegenstand von der Macht des Gemüts über seine krankhaften körperlichen Empfindungen" für sein Journal der praktischen Heilkunde (740). Joh. Friedr. Reichardt, der damals berühmte Komponist, wünscht für sein Journal "Deutschland" einen "den sittlichen Zweck und das innere Wesen der schönen Künste" betreffenden Beitrag (707). Biester gibt kurze Nachrichten über das Eingehen der Berliner Monatsschrift und will von Kant in den damals begründeten Berliner Blättern unterstützt sein (625, 732, 739; vgl. 766). Die italienische Akademie der Wissenschaften ernennt Kant zu ihrem Mitgliede und ersucht ihn, „de vouloir bien lui communiquer tous les deux ans quelque dissertation, pour l'insérer dans ses Mémoires dont elle publiera 1 Volume par an (765).

Die hohe Anerkennung, welche Kants System in Deutsclland, speziell Norddeutschland findet, lässt in anderen Ländern den Wunsch aufsteigen, die kritische Philosophie kennen zu lernen. Es werden Übersetzungen Kantischer Schriften in fremde Sprachen veranstaltet. So hören wir aus dem grösstenteils verloren gegangenen Briefwechsel Kants mit dem Buchhändler de la Garde $(623,697)$, dass ein gewisser Theremin [nicht der bekannte Kanzelredner und Homilet Theremin] an einer französischen Übersetzung der Kantischen Werke arbeitet. „Der hiesige Dänische Gesandtschaftsarzt," schreibt Kiesewetter aus Berlin, „der mein Zuhörer u. ein treflicher Kopf ist, wird Ihr Werkchen über den ewigen Frieden und [meine] Darstellung [Thres Systems] ins Dänische, und ein junger schwedischer Gelehrter, der auch meine Vorlesungen besucht, ins Schwedische übersetzen" (708). Der Würzburger Professor Matern 
Reuss hat "uber theor. Phlie ein Vorlesbuch in latein. sprache geschrieben, ${ }^{4}$ weil in Baiern, Schwaben und der katholischen Schweitz "die schulen meistens Von Mronchen besorgt werden, die aber nur nicht nach einem teutschen Vorlesbuch lesen dürfen, nach einem protestantischen (so sagen sie) gar nicht" (664). Aus einem Briefe L. H. Jakobs (712) erfahren wir, dass der Schottlander Richardson sich mit der Übersetzung der metaphysischen Anfangsgrïnde der Rechtslehre beschäftigt. Derselbe berichtet an Kant: „Unter dem gemeinen Titel: Versuche, habe ich viel metaphysische Materie versteckt. Durch. dieses Mittel hoffe ich meine Landsleute, die noch immer in der Empirie ersoffen sind, zu bewegen, dass sie eine besser gegründete, und nach meinem demüthigen Dafürhalten, die einzig wohl gegründete Philosophie studieren" (769). J. Glover fragt bei Kant an, ob er die "Metaphysischen Anfangsgründe der Natur Wissenschaft" ins Holländische übersetzen darf. Seinem Briefe gibt er eine beachtenswerte ${ }_{\text {nKurtze Übersicht der Förderungen und des Zustandes der Critischen }}$ Philosophie in der Batavischen Republik" bei (851). Ausser durch Übersetzungen sucht man die kritische Philosophie im Ausland durch Vorträge zu verbreiten. Doch hat man in Paris damit kein Glück gehabt, weil der Vortragende bloss die negative Seite des Kantischen Systems kannte. Nach Kiesewetters Bericht (788) „trug man vor einiger Zeit dem HE von Humboldt dem ältern auf, über die Resultate Ihres Systems im institut eine Vorlesung zu halten. Dieser unterzog sich auch dieser Sache, ob er gleich nicht das gehörige Zeug dazu hat und zeigte, der Nutzen der kritischen Philosophie sei negativ, sie halte die Vernunft ab, im Felde des Übersinnlichen Luftschlösser zu bauen. Die Pariser Gelehrten antworteten, dass sie nicht in Abrede sein wollten, dass Sie auf eine neue und scharfsinnigere Art die Wahrheit dieses Resultates bewiesen hätten, dass aber dadurch so viel eben nicht gewonnen sei, weil dis Resultat auch schon sonst bekannt gewesen, sie fragten, ob Sie denn blos eingerissen und nichts aufgebaut hätten, und denken Sie sich, Herr von Humboldt kannte blos den Schutt, der durch die Critik eingestürzten Systeme. Si tacuisset, philosophus mansisset."

Der gewaltige Erfolg der kritischen Philosophie ist es ferner, der illtere und jüngere Gelehrte veranlasst, die von ihnen verfassten Werke und Schriften Kant zur Prüfung und Beurteilung vorzulegen. Viele derselben sind erläuternde Auszüge aus Kants Werken oder wenigstens aus dem Geiste des Kritizismus erwachsen. Die Verfasser knüpfen daran häufig von Kant entweder unbeantwortet gelassene oder uns verloren gegangene Fragen nach dem Verständnis einzelner dunkler Stellen des Kantischen Systems oder nach wissenschaftlicher Aufklärung ïberhaupt oder geben ihrer gespannten Erwartung Ausdruck, diese oder jene im Druck befindliche Kantische Schrift bald erhalten zu können, oder sagen, dass sie mit Kants philosophischen Ansichten harmonieren, wobei sie über einzelne Kantische Gedanken und Schriften ihr Urteil abgeben. Sie alle gedenken gern der glückseligen Zeit, wo sie als treue Jünger Kant zu Füssen gesessen oder in persönlichem Verkehr mit ihm gestanden haben, und sind von tiefem Dank erfüllt für die mannigfachen geistigen Anregungen, die sie von Kant in der Nähe oder 
aus der Ferne erhalten haben. Hierher gehört der Briefwechsel mit dem Göttinger Kirchenhistoriker und Moraltheologen Stäudlin (616, 660, 700, 772, 790). Dieser, ein bewundernder Verehrer Kants, bittet um und dankt für Zusendung des Streits d. Fac. Sein Urteil über die ihm von Kant ebenfalls übersandte Schrift „Zum ewigen Frieden" lautet sehr optimistisch: "Sie wird die Aufmerksamkeit der Nationen einerndten und auf entfernte Geschlechter hinwirken. Fine lehrreiche Lection für Fürsten und Minister, sowie für. den Unterthanen, wird sie helffen, die Politik der Moral zu unterwerffen und die Menschen der brüderlichen Vereinigung näher zu bringen." - Chr. Friedr. Ammon aus Göttingen, einer der Hauptvertreter des theologischen Rationalismus, spricht den Wunsch aus: „Möchten besonders meine Ideen über die Wunder verdienen, von Ihnen geprüft zu werden, da es mir scheint, dass einige Ihrer Verehrer die Kritik der reinen Vernunft gar sehr zur Unterstïtzung ihrer mystischen Theorien von den Wundern missbrauchen" (665). Nach seiner Überzeugung „ist die Moraltheologie die einzig. wahre, reine, lebendige, und zugleich die Theologie Jesu und seiner Schüler." „Die Worte der Schrift: ich will mein Gesez in ihr Herz, und meine Kenntniss in ihren Verstand schreiben: enthalten die Basis aller, auch der chr. Offenbarung. An dieser unmittelbaren moralisch-religiösen Offenbarung müssen wir, dünkt mich, festhaiten, wenn nicht alle Religion zu Grunde gehen soll. Die mittelbare Offenbarung der Naturalisten (empirisch Rationalisten) in und durch die sichtbare Welt, scheint mir so gut, als gar keine, da es ihr an allen Principien der Moral und Religion fehlt" (771). - Fessler, der zur evangelischen Kirche ïbergetretene Jesuit und spätere evangelische Bischof von Neufinnland, möchte Kants Rat hören über die Art, wie er seinen Commentar uiber die Moralphilosophie der Stoiker, speziell Senecas einrichten und das Verhältnis Senecas zur kritischen Moralphilosophie bestimmen soll (634). Der Erlanger Theologe Seiler widmet Kant sein Buch von der Wahrheit des Christentums (6.24), und der Hallenser Privatdozent Morgenstern übersendet Kant, ebenfalls mit der Bitte um Beurteilung, sein Werk de Platonis republica, das Kants Anerkennuung findet (627, 637). - Gleichfalls aus Halle erhält Kant zur Prüfung ein populäres Buch über die kritische Religionstheorie, das Ludw. Heinr. Jakob zum Verfasser hat. „Um diesem Buche desto sicherern Eingang zư ,verschaffen," hat Jakob ,alle Betrachtungen iiber das Positive weggelassen und allein die reine Vernunftreligion dargestellt". "Um aber nicht ins trockene zu fallen, " hat er "den teleologischen Theil sehr weitläufig ausgeführt, es versteht sich, nicht als Beweis, sondern als Rührungs- und Belebungsmittel für den anderswodurch begriindeten Religionsglauben. Denjenigen," meint er weiter, "welche die kritische Philosophie in den übeln Ruf zu bringen suchten, dass sie die schönen und rührenden Betrachtungen der Natur aus der Religion entfernen wolle, ist nun der Mund gestopft" (712). -- Der Berliner Prediger Jenisch legt Kant sein das Ganze des kritischen Lehrgebäudes umfassendes Werk, dem von der Akademie der Wissenschaften das Accessit zuerkannt worden war, mit der Bitte um Durchsicht vor $(668,671)$. Das Werk soll „eine Darstellung, Erläuterung und Prüfung der Gründe und des Werts [des Kantischen] ganzen Lehrgebäudes seyn." Die von Bewunderung Kants überfliessenden 
Briefe sind wegen der an dem herrschenden philosophischen Geiste geiibten Kritik sehr beachtenswert. - Mellin wünscht ein Gutachten über sein bekanntes Encyklopidisches Worterbuch der kritischen Philosophie (672, 735, 760). Sein letzter Brief enthält einige interessante Gedanken über den dogmatischen Vortrag des Systems der Transscendentalphilosophie (819). - Der Kriminalist E. F. Klein schickt Kant sein Lehrbuch der natürlichen Rechtswissenschaft $z u$ und wünscht tiber eine wichtige kriminalrechtliche Frage Aufschluss: „Es füngt jetzt an, eine neuere Theorie im Criminalrechte Aufsehen $\mathrm{zu}$ erregen, nach welcher die Menschen bloss wie Thiere behandelt werden. Ich weiss wohl, dass die Freyheit des Willens nicht sinnlich wahrgenommen werden kann; aber eigentliche Strafe setzt doch den Fall voraus, wo der Mensch nicht bloss als Pflanze oder Thier wirksam gewesen ist" (681). Eine Antwort Kants kennen wir leider nicht. - Von dem Stabskapitän v. Stårck, dessen Brief (687) eine mathematische Beweisführung in sich schliesst und deshalb für Mathematiker von Interesse ist, werden Kant einige mathematische Aufsätze und von dem Regierungsrat Dannenberg aus Posen eine Reihe philosophischer Gedanken zur Beurteilung vorgelegt (683). - G. B. Jäsche überreicht den Versuch eines moralischen Katechismus, d. i. ein populäres Lehrbuch der Rechts- und Pflichtenlelire und erwartet von Kant ein Urteil darüber, ob ein encyklopädisches Lehrbuch für die wissenschaftliche Kultur überhaupt vorteilhaft sein könne (684). - Der schon genannte Hufeland unterbreitet Kant einen Versuch, „das Physische im Menschen moralisch zu behandeln, den ganzen, auch physischen Menschen als ein auf Moralität berechnetes Wesen darzustellen, und die moralische Kulturals unentbehrlich zurphysischen Vollendung der überall nur in der Anlage vorhandenen Menschennatur zu zeigen" (693) Kant will diese „kühne aber zugleich seelenerhebende Idee von der selbst den physischen Menschen belebenden Kraft der moralischen Anlage in ihm" sich klar machen und sie auch für die Anthropologie benutzen (704). - Der dreiundzwanzigjährige Christian Weiss will wissen, ob er den Geist der kritischen Lehre getroffen hat. „Geht die Wahrheit und das Leben aus den Objekten in uns? oder leiht nicht vielmehr der Geist den Dingen ausser ihm (welche ohne ihn Nichts sind,) das eine wie die andere?" (727). - Der Pfarrer Möller zu Volkenroda bittet um Kants Urteil über eine seiner "Lieblingsideen", nämlich darüber, „ob wohl im Bewusstseyn Grade sich denken liessen (731). - Christian Garvè widmet Kant, die seine verdienstliche Übersetzung der aristotelischen Ethik einleitende „Übersicht der vornehmsten Principien der Sittenlehre von Aristoteles bis auf unsere Zeit" (779). Diese Abhandlung soll dazu bestimmt sein, die "verborgene und stillschweigende Verbindung, welche schon lange unter uns vorhanden ist, gegen das Ende unseres Lebens noch fester zu knüpfen" (780). In der Antwort Kants findet sich ein wichtiger Protest gegen "die Note S. 339“ des übersandten Buches: „Nicht die Untersuchung vom Daseyn Gottes, der Unsterblichkeit ist der Punkt gewesen, von dem ich ausgegangen bin, sondern die Antinomie der r. V. . . . diese.war es, welche mich aus dem dogmatischen Schlummer zuerst aufweckte und zur Critik der Vernunft selbst hintrieb, um das Scandal des scheinbaren Widerspruchs der Vernunft mit ihr selbst zu heben" (781). Aus demselben Briefe 
hören wir, dass Kant sich mit dem „Übergang von den metaphys. Anf. Gr. D. N. W. zur Physik" beschäftigt (Sept. 1798). - F. E. Reichsgraf zu Dohna wïnscht ebenso wie der Hallenser Professor der Medizin Juncker Auskunft darüber, ob und inwiefern Kant die Einimpfung der Blattern für sittlich oder unsittlich halte $(803,825)$. - Schliesslich ist unter dieser Rubrik noch zu nennen R. Marquis de Mesmon, der Kant den Prospekt eines von ihm verfassten Buches (764), und J. M. C. Baron von Utenhove, der Kant ein Exemplar der von ihm übersetzten Lambertschen Cosmologischen Briefe (847) zur Beurteilung vorlegt.

Die hohe Verehrung, die Kart gegen Ende seines Lebens geniesst, schliesst nicht aus, dass sich hier und da Einwände gegen die kritische Philosophie erheben. Nicht ïberall bricht sich die Philosophie Kants Bahn. Wir hören ausser von ihren Erfolgen auch von Hemmungen, auf die sie stösst Wir haben eine Reihe von Briefen, die uns einen Einblick in diese Entwicklung verschaffen. Günstig in dieser Hinsicht lauten die Nachrichten, die Reinhold, der durch seine „Briefe über die Kant'sche Philosophie" verdiente Kantianer, durch den von Kiel nach Königsberg übersiedelnden jungen Grafen von Purgstall an Kant bringen lässt: auch in Kiel hat „das Evangelium der praktischen Vernunft nicht weniger als in Jena Eingang gefunden (620). Kants kurze Antwort. (633) ist in der 2. Hartensteinschen Ausgabe abgedruckt. - Günstig lautet auch der Bericht Ammons hinsichtlich der Göttinger Verhältnisse. Er schreibt zunächst von sich selbst: „Ich bin nach einem unbefangenen Studium Threr vortreflichen Werke vollkommen überzeugt, dass die Theologie durchaus keine sichere Haltung hat, wenn sie nicht auf einen moralischen Grund gestüzt wird:" Nach einer kurzen Reproduktion der Prinzipien derKantischen Glaubenslehre heisst es dann weiter gegen den Schluss des Briefes hin: „Es ist traurig genug, ‘dass man hie und da - denn hier in Gọttingen haben wir freie Hand - nicht einsehen will, dass nur anf diesem Wege eine feststehende Religionslehre gefunden und den Pseudo-Theologen unserer Zeit entgegen gearbeitet werden kann, die durch ihre einseitigen Aufklärungen es auf nichts Geringeres, als auf den Ruin aller systematischen Theologie angetragen haben. Schon sind Ihre Grundsätze, grosser Lehrer, unter unseren besseren Theologen zu allgemein, als dass ein plötzlicher Stillestand zu befürchten wäre; sie werden zum Segen für die Menschheit wuchern und Früchte tragen für die Ewigkeit" (626). Drei Jahre später, im Aug. 1798, berichtet derselbe aus Göttingen: „Der Sieg der kritischen Philosophie, besonders von ihrer praktischen Seite, wird auch auf unserer Akademie immer entscheidender. Vergebens bieten die sophistische Gnosis und die Gewalt des Buchstabens ihre Kräfte gegen sie auf. Das Studium derselben bekommt dadurch nur neues Leben, und ihre Erkenntniss mehr Gründlichkeit und eine grössere Reinheit als auf den Universitäten, wo man sie mit einem kritisch scheinenden Scholasticismus zu verbrillantiren sucht" (774). - Sehr gemischt lauten die Nachrichten über „den Zustand der kritischen Phlie im katholischen Deutschland" (664) und in Österreich. Aus einem Briefe des Würzburger Professors Matern Reuss vom April 1796 hören wir, dass in Würzburg in der Philosophie, Theologie und der Rechtswissenschaft nach Kantischen Grundsätzen gelehrt wird und solche auch 
dem Religionsunterrichte und der Predigt zugrunde gelegt werden. „Nicht gar so hell, doch ziemlich hell sieht es auf den hohen schulen Bamberg Heidelberg und andern katholischen Schulen aus, desto finsterer ist es áber in Baiern, schwaben und der katholischen schweitz" (664). - Ergänzt wird dieser Brief durch einen solchen des W iirzburger Dozenten Konrad Stang (680). „Die kritische Philosophie ist in der üsterreichischen Monarchie als Feindinn erklurt, und wehe dem, der sie lehren will. Der Kaiser ist ganz dagegen eingenommen, und da ihm der Direktor der Schulen und des Studiums in Wien H. v. Birkenstock das kritische Sistem anpriess, so drehte sich der Kaiser herum und sagte: ich will einmal für allemal von diesem gefährlichen Sisteme nichts wissen." Zwei Männer haben ihr Lehramt verloren, weil sie im Geiste der kritischen Philosophie lehrten. In Wien, meint Stang, „wird nie viel zu Stande kommen, da es ganz an gelehrtem Gemeingeiste fehlt und die Professoren an der Universität einander nicht kennen: denn es ist reiner Zufall, der hier einen oder andern zusammenführt. In Salzburg geht es schon besser 'mit der kritischen Philosophie: besonders verwendet sich der würdige Regent des Priesterhauses dafür." „In München ist an keine kritische Philosophie zu denken, da Stattler hier wohnt und regiert." Derselbe Brief enthält ausser diesem Bericht noch Gedanken über das Naturrecht und einige die Schlacht bei Würzburg betreffende Kriegsneuigkeiten.

Der vorliegende Briefwechsel macht uns nun noch mit einzelnen Gegnern der kritischen Philosophie persönlich bekannt. Da ist zunächst der originelle, vor hundert Jahren verstorbene Arzt, Mystiker und Pietist Samuel Collenbusch. Dieser findet an Kants Moralphilosophie - manches zu tadeln (vgl. 517 im II. Bd.). "Ich habe mir,“ so schreibt er an Kant, „diesen Sommer Ihre Morall und Religion ein par mahl Vorlesen lassen, Ich kan mich nicht überreden Dass es Ihnen ein Ernst sein solte, Was Sie Da Geschribem Habem, Ein Von aller Hoffnung gantz reiner Glaube, und ein Von aller Liebe gantz Reine Morall, Dass ist eine seltsame Erscheinung in Der Repuplick Der Gelehrten." "Ich Verkauffe meine Hoffnung nicht für Taussend Tonnen Goldes." Diesem Briefe folgt im März 1795 eine briefliche Auseinandersetzung Collenbuschs über den Unterschied von Wissen und Wollen, Können und Thun, und ein Jahr später plagt ihn der Gedanke, „was die ursache sein mag das mein Vernünftiger Bruder, Immanuel Kant nicht ebenso wohl als ich, sich darüber freuen kan, oder sich nicht darüber freuen will, dass Gott so gütig ist als Er ist." Eine Erwiderung Kants kennen wir leider nicht. Es wäre interessant gewesen zu sehen, wie Kant den teilweise berechtigten Vorwürfen gegenüber sich verantwortet hätte. - Philosophisch wichtiger ist der Brief eines anderen Gegners der kritischen Philosophie, nämlich der des Franzosen Sylvestre Chauvelot (685). Der Eingangssatz zeigt, um was es sich handelt: „Raisonnons un moment, vous et moi, en philosophes; en philosophes amis de la raison, de la sagesse, de la moralité, de la vraie perfectibilité de l'homme . . . Chauvelot ist ein begeisterter Freund jenes Gottesbeweises, der sich auf die sinngemässe Betrachtung der Welt grïndet, speziell die Astronomie zur Grundlage hat: „.. de toutes les Sciences que nous acquérons par les lumieres de la Nature, il n' en est 
aucune qui, mieux que l'Astronomie, nous mene à la connoissance d' un Etre souverain et tout parfait, qui nous fournisse des preuves mieux senties de son Existence, et qui mette dans un plus grand jour sagesse, sa bonté, et sa puissance infinie." In diesem Sinne lässt Chauvelot den alttestamentlichen David (Psalm 19,1), ferner Cicero, Descartes und Newton sprechen. Im Anschluss an diese Auseinandersetzungen werden andere interessante Fragen erörtert, z. B. „la Question qu' on a si souvent agitée: Si une societé d' Athées pourroit long-tems subsister?" Sie wird auf grund historischer Thatsachen verneinend beantwortet. Die letzten Seiten des Briefes beschäftigen sich mit der "Perfectibilité de l'homme." "Vous fondez vos espérances sur la Perfectibilité de l'homme, sur le progrès et le développement universel des lumieres qui s'avancent à grands pas, et dont l' effet d'après votre calcul, doit entraîner un jour jusqu' à la surpression des Lois." "Cette idée est un Rêve et ce Rêve est celui du bon Abbé de Saint-Pierre, qui, dans son systême de la paix universelle, vouloit ainsi faire fraterniser entr' eux tous les Souverains et tous les Peuples". „Perfectibilité ne peut et ne doit signifier autre chose que tendance à la perfection."

Sehen wir nun einmal von der direkten Beziehung; welche der vorliegende Briefwechsel auf Kant hat, ab, so verlieren die Briefe doch keineswegs jegliches Interesse. Was den Briefwechsel trotzdem so ausserordentlich interessant. macht, sind die ungemein zahlreichen Urteile über damals lebende Personen und Verhältnisse. Einiges davon haben wir schon gehört. Ich weise noch in Kürze auf die Briefe hin, die in dieser Hinsicht für die Philosophie- und Kulturgeschichte von Wichtigkeit sind. Hierher gehört der umfangreichste Briefwechsel des ganzen Bandes, nämlich der mit Kiesewetter. Im Ganzen 20 Briefe, von denen 11 Kant zum Verfasser haben. Von diesen 11 sind 4 nicht mehr erhalten, 2 (782 und 827) sind in der 2. Hartensteinschen Ausgabe abgedruckt (vgl. auch Sintenis in der Altpreuss. Monatsschrift XV, 1878). Persönliches von ${ }_{a}^{\mathrm{g} K i e s e w e t t e r, ~ d a n n ~ l i t e r a r i s c h e, ~ p o l i t i s c h e ~ u n d ~ B e r l i n e r ~ N e u i g-~}$ keiten, ferner Neuigkeiten aus dem beiderseitigen Bekanntenkreise, dazwischen Berichte über die Aufnahme der kritischen Philosophie bilden vor allem den Inhalt der Briefe, speziell von Seiten Kiesewetters. Einen breiten Raum nehmen bei Kiesewetter Mitteilungen über Veränderungen seiner Lebenslage ein. Seinen Entschluss, „die litterärische Laufbahn ganz zu verlassen, dafür aber in [die] von der Accise überzugehen," nimmt Kant mit Befremden auf (878). Weiterhin hörén wir, dass Kants Schrift über den ewigen Frieden von. Kiesewetter kommentiert wird, und dass in mehreren Klöstern über Kiesewetters, nach ${ }_{\varsigma}^{5}$ Kantischen Grundsätzen abgefasste Logik Vorlesungen_gehalten werden. Was uns nun aber besonders interessiert, sind zwei scharfe Urteile Kiesewetters, das eine über Herder, das andere über Nikolai. Herder hatte sich, erbittert über das wachsende Ansehen der Kantischen Philosophie, zu heftigen Angriffen in seiner „Metakritik" hinreissen lassen. Kiesewetter hält „das Herdersche Geschwätz an sich kaum einer Widerlegung würdig," und "ich würde," so schreibt er, "mich auch nicht damit befasst thaben, wenn"der alte radottirende Wieland im Deutschen Merkur nicht so gewaltig zum Lobe dieses Geschreibsel in 
die Posaume gestossen hilte und der Ton des sonst so gleisnerischen, pfäfischen Herders mich nicht so schr beleidigt hatte." "Nichts hat mich mehr amusiert, als wenn Herder über Mathematik zu schwatzen anhebt; es ist kaum möglich, weniger als er in den Geist dieser Wissenschaft eingedrungen zu sein und doch arroganter. darüber zu sprechen" (809). Über den Buchhandler und Kritiker Nikolai heisst es in demselben Briefe: "Nikolai phantasiert noch immer iiber kritische Philosophie und Fichtianismus; und nun er Academicien geworden, hält er es für Pflicht, sein Geschreibsel zu verdoppeln." Interessant ist noch, aus diesen Briefen zu erfahren, dass Kiesewetter Sonntags über Kants Anthropologie in einem von Personen aller Stände vollbesetzten Hörsaale Kolleg liest. In fast allen Briefen finden sich ausserdem Notizen über die Zusendung, Verpackung und Zubereitung der Teltower Rübchen, deren alljährliche Lieferung an Kant Kiesewetter übernommen hat. - Abfällige Urteile über Reinhold, Fichte, Abicht u. a. und über „Klopstocks Ausfall auf die Kritik" enthalten die Briefe des Hallenser Professors L. H. Jakob. Dieser trägt sich, wie wir ausserdem hören, mit dem Plane, eine Biographie Kants zu schreiben, und sucht eine Professur in Göttingen, zu deren Erlangung ihm Kant durch Empfehlung behilflich sein soll. - Der kulturgeschichtlich interessante Briefverkehr Kants mit dem Berliner Prediger Lüdeke (von Kant 759, von Lïdeke 754, 763; 785, 791) fällt in die erste Regierungszeit Friedr. Wilh. III., über den Lüdeke manches mitteilt. „Unser lieber junger König erhebt unser Herz mit herrlichen Ioffnungen." "Unter dem 27. Dez. hat das Ober Consistorium alle ihm geraubten Rechte der Examination, Censur etc. wieder bekommen und mithin wird wohl die Glaubens Comission wie die Tobacksfirma aufgehoben sein. Ach es wird einem so wohl, wenn der Nebel gefallen ist und die Sonne sichtbar und wirksam wird. Nun wird auch wohl selbst die Religion innerhalb der Grenzen der Vernunft durch die Censur kommen können"(754). Später: „Unser lieber König fährt fort, für das allgemeine Beste zu sorgen. Privathäuser bauet er nicht: aber dagegen Chaussees, Promenaden und lässt die Strassen gehbar machen, auf welchen man sonst Hals und Bein ganz bequem brechen konnte." „Der Studirwuth scheint er auch Einhalt thun zu wollen" (785). Die Briefe Liidekes sind nicht ohne Humor: „Ein Politiker", so schreibt er, „bin ich so wenig, dass ich manchesmahl in acht Tagen keine Zeitungen lese, weil ich das Lügen nicht liebe. Ein Philosoph bin ich auch nur so für das Haus und vor allem Predigen und Krankenbesuchen kann ich kaum meine Freunde mit Versen plagen" (785). "Mein Glaubens Bekenntniss ist dieses: Ohne Vernunft Gebrauch Theologe seyn sollen, kommt mir vor als unter der ausgepumpten Glocke der Luftpumpe athmen und singen sollen. Das können doch höchstens nur Frösche" (791). Kants einmalige Antwort enthält nichts Bemerkenswertes. - Der schon erwähnte Joh. Richardson sieht in Fichtes Philosophie "absurde Theorien" und "ungeheure Verirrungen" (769, 770). - Sehr lesenswert sind die Briefe J. H. J. Lehmanns; dieselben sind ausserordentlich reich an Urteilen über die verschiedensten Göttinger Gelehrten und über die Göttinger Universitäts- und Fakultätsverhältnisse (793, 794, 808, 813). - Nachrichten über das Gymnasium in Mitau und die Universität Dorpat enthalten die beiden Briefe C. W. Cruses 
(783, S05). Dieser bittet um ein akademisches Fleisszeugnis und um Mitteilung der Bedingungen, unter welchen er abwesend den gradum magistri philosophiae erhalten kann. Ebenso wünscht der Dorpater Professor der Cameralwissenschaft und Statistik C E. Chr. Müller, ohne anwesend zu sein, „gegen Erlegung der erforderlichen Kosten ein Doktor- oder Magisterdiplom" ausgefertigt zu haben, da er nur als Doktor oder Magister eine Professur in Dorpat verwalten kann (833).

Mit dem, was bisher unter bestimmten Gesichtspunkten angeführt oder bloss angedeutet ist, ist der bedeutsame Inhalt des vorliegenden Briefwechsels noch nicht erschöpft. Zwar das Wichtigste, was noch fehlt, besonders von Seiten Kants, ist a us der Hartensteinschen Ausgabe bekannt: Der Briefverkehr mit Schiller, Soemmering, Erhard, Schütz, Lindblom, Herz, Lichtenberg und Richter bedarf daher keiner Erwähnung mehr. Dagegen müssen andere Briefe noch herangezogen werden.

Interessant z. B. ist es, aus drei längeren Briefen Reinh. Bernh. Jachmanns zu erfahren, dass dieser seine Predigten „stets nach den Grundsätzen der reinen Sittenlehre abfasse." Dieselben Grundsätze will er dem Katechismusunterricht zugrunde gelegt wissen. Dann „würde nicht allein der Inhalt der christlichen Lehre mehr Autorität erlangen, da man sähe, dass ihre Lehren mit den reinen Vernunftlehren übereinstimmen, sondern es würden auch überhaupt alle die Zweifel und Irrtümer wegfallen, die bei den theoretischen Beweisen von Gott, Freyheit und Unsterblichkeit unvermeidlich sind. Die Freyheit des Willens würde sich als ein Factum der Vernunft aufdringen und Grott und eine künftige Fortdauer würden ihm Vernunftbedürfnisse seyn, an welche ihn ein Vernunftglaube fesselt, den keine spekulation wankend zu machen vermag." Der.Brief vom Okt. 1797 enthält persönliche Angelegenheiten Jachmanns. Er wünscht in Königsberg eine Prediger- und daneben eine Dozentenstelle in der philosophischen Fakultät $z u$ bekleiden. Seinem letzten, vom Aug. 1800 stammenden Schreiben hat er Fragebogen beigelegt, die auf eine Biographie Kants abzielen. Von Seiten Kants ist nur ein unbedeutendes Fragment vorhanden.

Philosophierender Natur sind die Briefe Becks an Kant (vgl. Altpreuss. Monatsschr. XXII, 1885 und Archiv f. Gesch. d. Philos. II, 4, 1889). In den beiden umfangreichsten Briefen vom 20. und 24. Juni 1797 legt Beck die Hauptpunkte seiner transscendentalphilosophischen Ansichten dar; die Briefe bilden die Verantwortung auf die ihm vom Hofprediger Schultz gemachten, auf angeblichen Umsturz der kritischen Philosophie sich beziehenden Vorwürfe. Hier und da kommt deshalb ein gereizter Ton gegen Schultz zum Vorschein. Kant wünscht in einem Briefe an Tieftrunk (725), dass Beck „diesen Ton bey Gelegenheit in den Ton der Freundschaft umstimmen möchte; denn was sollen uns," sagt Kant aus der ihm eigenen vornehmen Sinnesart heraus, "alle Bearbeitungen und Streitigkeiten der Speculation, wenn die Herzensgüte darüber einbüsst?"

Lesenswert sind die beiden eigenartigen Briefe eines Joh. Plücker aus Elberfeld $(656,662)$, der ,von Jugend auf, jetzt in die 60 alt syende, 
sich nach Wahrheit umgesehen" und nun sich in Hoffnung auf Belehrung mit den charakteristischen Worten an Kant wendet: „Übel Werden Sie's mir doch nicht nehmen!" „Sic gaben mir den Schlilssel - zur Erkäntniss der tiefen Weissheit - die Jesus Christus - durch seine Lehre und Reden ge'lussert." Kant, dem dies Schreiben „eine angenehme Stunde gemacht" hat, ilussert sich in seiner Antwort u. a. über die, welche „die einfachste Sache von der Welt geflissentlich $\mathrm{zu}$ der schwierigsten machen, indem sie, wie Ärzte, in Recepten, des guten nicht zuviel thun zu können wälnnen, und die moralisch Kranken mit Glaubensvorschriften überfüllen, bis ihnen darüber der Geist (das wahre Prinzip der guten Deutungsart) ausgeht" (657).

Nur ganz Persönliches enthalten die zahlreichen Briefe Friedr. A ug. Hahnrieders, der, von "subtil ausgedachten Scrupeln und moralischen Bedenklichkeiten" abgehalten, ein staatliches Amt zu übernehmen, nur im selbständigen Handwerk, später in der selbständigen Bewirtschaftung eines Landgutes glïcklich $\mathrm{zu}$ werden hofft.

Der Greifswalder Theologe Gottlieb Schlegel gibt dem moralischen Vernunftgebot die Form: „Handle nach dem Ausspruch der Vernunft, zufolge einer lautern Betrachtung der Dinge;" weiterhin wünscht er von Kant darüber belehrt zu werden, ob „die Untersuchung der Gewissheit der Erkenntniss auf das Daseyn Gottes führe, und der Glaube an Gott zur Beruhigung in Anselung der Ungewissheit des menschlichen Wissens beytrage" (673). - Von religionsphilosophischem Interesse ist auch der Brief des Predigers Dominici aus Oels, der über eine Dunkelheit in Kants moralischem Gottesbeweis aufgeklärt $\mathrm{zu}$ sein wünscht; er meint nicht zu irren, wenn er den moralischen Beweis durch folgenden Syllogismus ausdrückt: „Wenn kein Gott ist: 'So ist die Ausübung des moralischen Gesetzes (weil alsdann keine der moralischen guten Gesinnung angemessne Glückseeligkeit zu hoffen ist) unmöglich. Nun ist das zweyte falsch. Also auch das Erste." Einem zweiten Syllogismus gibt er die folgende Form: „Der Mensch kann nicht ein Wesen seyn, welches unauflösliche Widersprüche enthielte. Ein solches würde er aber seyn, wenn keine Unsterblichkeit wäre. Also ist eine Unsterblichkeit. Sie kann aber olne Gott nicht seyn. Also ist ein Gott."

Die philosophisch wichtigen Briefe an Tieftrunk sind aus der Hartensteinschen Ausgabe grösstenteils bekannt. Es kommen hinzu von Kants Seite 725 und 746, aus Tieftrunks Feder 718, 748, 824, ausserdem 3, von denen wir nur das Datum wissen. Den grössten philosophischen Wert von diesen Briefen hat das grosse Brieffragment Tieftrunks vom 20. Juni 1797; dasselbe behandelt ausführlich einige Fragen aus der Kategorienlehre und erörtert dann den Unterschied zwischen Anschauung und Denken.

Was von dem Briefwechsel mit Wilmans erhalten ist, bietet wenig Bemerkenswertes, da der Brief 711 schon aus dem Streit d. Fac. bekannt ist. In dem Briefe vom Mai 1799 geht Kant ganz kurz auf die ihm unverständliche Behauptung Wilmans', "dass zwischen Vernunft und Verstand ein gänzlicher Unterschied, der letztere aber ein blos materielles Wesen sey," ein (800). 
Von der physikalischen Erscheinung, „dass geschmolzenes Kupfer über Wasser gegossen darüber ruhig starr werde, dahingegen Wasser über geschmolzenes Kupfer gegossen, dieses gänzlich zersprengen wercle," handelt der einmalige Briefverkehr Kants mit C. G. Hagen $(816,817)$. Der grösstenteils verloren gegangene Briefwechsel mit Rink berichtet, von einem Streit Rinks mit dem Buchhändler Vollmer, die Herausgabe von Kants Physischer Geographie betreffend $(798,802,853,855)$. Auf diesem Streit bezieht sich die öffentliche Erklärung No. 7. - Für den Kandidaten der Mathematik Lehmann verwendet sich Kant nicht bloss bei dem Oberschulrat Meierotto, sondern auch bei dem Regierungspräsidenten v. Massow (Entwurf 729 und 730). - Von Vigilantius erbittet sich Kant in einigen rechtlichen Fragen Aufschluss (761, 839). - Verlegersachen behandelt der Briefwechsel mit Nicolovius (637, 638, 728, 768, 814, 815). Eine Reihe von Briefen betrifft oder enthält beiläufig Nachrichten über die Zusendung von leiblichen Bedürfnissen an Kant: genannt sind Teltower Rübchen (in den Briefen Kiesewetters), Göttinger Würste; Obst (645, 793, 794, 808, 813), Linsen und Bohnen $(651,669)$, Offenbacher Schnupftabak (787, 645), Ungarwein (854).

Familiären Charakter trägt der Briefverkehr Kants mit seinen Verwandten. Die Kinder seines Bruders Joh. Heinr. Kant bitten in rührenden Worten den Onkel um eine Locke aus seinem ehrwürdigen grauen Haar, da es ihnen nicht, vergönnt sei, ihn persönlich kennen zu lernen. Maria Kant geb. Havemann erhält nach dem Hinscheiden ihres Mannes, eines kurländischen Geistlichen, für sich und ihre unversorgten Kinder eine vierteljährliche Unterstützung $(821,829)$, nachdem sich ihr Schwiegersohn, der Predigtamtskandidat Schoen für sie bei Kant verwandt hatte $(828,852)$. Auch sonst unterstützt Kant seine Verwandten (695). Um Geldunterstützung scheint er oft angegangen zu sein $(710,720)$. Der Brief Kants aus dem Jahre 1803 besteht in einem Glückwnnsch zur Verlobung seiner Bruderstochter mit Fr. Stuart.

Unter den undatierten Briefen ist nur der Brief von Jurgulan (861) von Interesse. Gegenstand dieses Briefes bildet das „Gespräch eines Heydnischen Printzen von der Christlichen und seiner Heydnischen Religion."

An den Briefwechsel schliessen sich die öffentlichen Erklärungen, die mit Ausnahme der ersten unwichtigen alle aus der Hartensteinschen Ausgabe bekannt sind.

Die handschriftlichen Erklärungen beginnen mit „Entwürfen in einer Streitsache mit Carl Georg Burckhardt" aus den Jahren ca. 1784-1786; die Entwürfe betreffen Mietskontraktsfragen. No. 2 bezieht sich auf die Cabinetsordre König Friedr. Wilh. II. No. 3, die „Rechtfertigung des Directoriums der französischen Republick" ist aus der Hartensteinschen Ausgabe bekannt. Es folgt dann unter 4 Kants Testament, unter 5 Bestimmungen über sein Begräbnis, unter 6 eine Ergänzung zum letzten Willen und unter 7 die Verschenkung der goldenen Kant-Medaille an den Diakonus Wasiansky. 
110 E. Sänger, Die neue Kantausgabe: Kants Briefwechsel.

Von den Denkversen zu Ehren verstorbener Collegen ist der Ehrendenkspruch auf den am 21. Juni 1780 verstorbenen Rektor und Professor der prakt. Philosophie C. A. Christiani noch nicht bei Hartenstein abgedruckt.

Es folgen 3 Kant von seinen Zuhörern gewidmete Gedichte und 10 Stammbuchverse, 7 lateinische und 3 deutsche. Der Band schliesst mit einer Auswahl des amtlichen Schriftverkehrs, der uns einen Blick in die Behandlung rechtlicher Universitäts- und Fakultätsfragen verschafft. 